\title{
Controller to Avoid Bifurcations of Stable Fixed Point Using Spectral Radius Optimization
}

\author{
Ken'ichi Fujimoto
}

\begin{abstract}
The dynamics of systems in the real world can be defined by differential or difference equations. In many dynamical systems, the appearance of a stable fixed or periodic point corresponding to the desired behavior means that the system works well. Stable one can be bifurcated with unexpected change of system-parameter values, and an in appropriate bifurcation can cause system down. This paper presents a novel controller to avoid inappropriate bifurcations of stable fixed points in discrete-time dynamical systems. The controller is designed on the basis of optimizing an objective function with the spectral radius of the Jacobian matrix for a target stable fixed point. Numerical control experiments for a stable fixed point in the Hénon map are also demonstrated to show the effectiveness of the proposed controller.
\end{abstract}

Keywords - Discrete-time dynamical system, stable fixed point, bifurcation avoidance, Hénon map, Jacobian matrix, spectral radius.

\section{INTRODUCTION}

In nonlinear dynamical systems represented by parameterised differential or difference equations, we set system parameters to appropriate values so that desired behavior can appear in a steady state. However, when unexpected parameter change occurs for any reason, a bifurcation [1] of the desired behavior can occur and inappropriate behavior can appear, i.e., the system may not work as expected. For example, alternans, which is a deadly heart disease, is caused by a period-doubling bifurcation of behavior corresponding to a normal state [2].

Fujimoto et al. [3, 4] have previously proposed a parametric controller to avoid inappropriate bifurcations of stable fixed or periodic points in discrete-time dynamical systems for unexpected parameter change on the basis of minimization of the maximum local Lyapunov exponent. Moreover, using a stroboscopic mapping that transforms the trajectory of a continuous-time periodic solution into a sequence of points, Fujimoto et al. [5] have proposed a controller for stable periodic solutions in continuous-time dynamical systems with periodic external input.

In this paper, I propose another approach based on minimizing an objective function with the spectral radius of the Jacobian matrix for a stable fixed point. I show that the gradient of the spectral radius needed in its minimization can be calculated by the formu la [6] and the differential of solution to the dynamical system with regard to a parameter. Moreover, I demonstrate numerical control experiments for a stable fixed

Ken'ichi Fujimoto, Kagawa University, Japan

E-mail:kfujim@cc.kagawa-u.ac.jp point observed in the Hénon map [7] to show the effectiveness of the proposed controller.

\section{PRELIMINARIES}

\section{A. Fixed Point and its Stability}

I consider the discrete-time dynamical systemdescribed by

$\boldsymbol{x}(t+1)=\boldsymbol{f}(\boldsymbol{x}(t), \boldsymbol{a}(t), \boldsymbol{b}(t))$

where $t$ is the discrete time, $\boldsymbol{x}(t) \in \boldsymbol{R}^{N}$ is the vector of state variables. $\boldsymbol{a}(t) \in \boldsymbol{R}^{M}$ is the vector of time-variant system parameters, which their values can be forcibly changed for any reason and are out of control. $\boldsymbol{b}(t) \in \boldsymbol{R}^{L}$ is also the vector of time-variant system parameters and are able to be handled. $N$, $M$, and $L$ correspond to the number of state variables and system parameters. I here assume that the change of these system parameters is significantly slow compared with those of the state variables.

At certain parameter values $\boldsymbol{a}^{*}$ and $\boldsymbol{b}^{*}$, the point $\boldsymbol{x}^{*}$ satisfying

$$
\boldsymbol{x}^{*}-\boldsymbol{f}\left(\boldsymbol{x}^{*}, \boldsymbol{a}^{*}, \boldsymbol{b}^{*}\right)=\mathbf{0}
$$

is called a fixed point. Its topological characteristic is defined with the positions of roots $\left(\mu_{i} s\right)$ of the equation defined as

$$
\chi\left(\mu_{i}\right)=\operatorname{det}\left(\mu_{i} \boldsymbol{I}-\left.\frac{\partial \boldsymbol{f}\left(\boldsymbol{x}, \boldsymbol{a}^{*}, \boldsymbol{b}^{*}\right)}{\partial \boldsymbol{x}}\right|_{\boldsymbol{x}=\boldsymbol{x}^{*}}\right)=0
$$

where $\boldsymbol{I}$ is the identity matrix with the size of $N \times N$. The roots corresponding to the eigenvalues of the Jacobian matrix are called characteris tic multipliers.

The point $\boldsymbol{x}^{*}$ is stable only if all the eigenvalues are inside the unit circle on the Gauss plane. Bifurcations of $\boldsymbol{x}^{*}$ occur when one or more eigenvalues are on the circumference of the unit circle, i.e., when the spectral radius of the Jacobian matrix defined as

$$
\rho\left(\boldsymbol{D}^{*}\right) \equiv \max _{i}\left(\left|\mu_{i}\right|\right)
$$

becomes one, where $\boldsymbol{D}^{*}=\partial \boldsymbol{f}\left(\boldsymbol{x}, \boldsymbol{a}^{*}, \boldsymbol{b}^{*}\right) / \partial \boldsymbol{x}$ at $\boldsymbol{x}=\boldsymbol{x}^{*}$.

\section{B. Gradient of Spectral Radius}

I now assume that $\rho\left(\boldsymbol{D}^{*}\right)$ is differentiable with regard to 
state variables and system parameters. I also assume that two eigenvectors, $\boldsymbol{u}$ and $\boldsymbol{v}$, are normalized as $\overline{\boldsymbol{u}}^{\mathrm{T}} \cdot \boldsymbol{v}=1$ where $\boldsymbol{u}$ is a left eigenvector of the eigenvalue with the maximum magnitude $\hat{\mu}, \overline{\boldsymbol{u}}$ represents the complex conjugate of $\boldsymbol{u}$, the superscript symbol T denotes the transpose of a vector, and $v$ is a right eigenvector of $\hat{\mu}$.

Using a differentiation formula on the spectral radius of a matrix [5], I derived a formu la on the gradient of $\rho\left(\boldsymbol{D}^{*}\right)$ with regard to $b_{\ell} \in \boldsymbol{b}$ as follows:

$$
\begin{aligned}
& \frac{\partial \rho\left(\boldsymbol{D}^{*}\right)}{\partial b_{\ell}} \\
& =\frac{\mathfrak{R}(\hat{\mu})}{|\hat{\mu}|}\left(\mathfrak{R}(\boldsymbol{u})^{\mathrm{T}} \frac{\partial \boldsymbol{D}^{*}}{\partial b_{\ell}} \mathfrak{R}(\boldsymbol{v})+\mathfrak{T}(\boldsymbol{u})^{\mathrm{T}} \frac{\partial \boldsymbol{D}^{*}}{\partial b_{\ell}} \mathfrak{T}(\boldsymbol{v})\right) \\
& +\frac{\mathfrak{T}(\hat{\mu})}{|\hat{\mu}|}\left(\mathfrak{R}(\boldsymbol{u})^{\mathrm{T}} \frac{\partial \boldsymbol{D}^{*}}{\partial b_{\ell}} \mathfrak{I}(\boldsymbol{v})-\mathfrak{J}(\boldsymbol{u})^{\mathrm{T}} \frac{\partial \boldsymbol{D}^{*}}{\partial b_{\ell}} \mathfrak{R}(\boldsymbol{v})\right)
\end{aligned}
$$

where $\mathfrak{R}(\cdot)$ and $\mathfrak{I}(\cdot)$ correspond to functions that return the real and imag inary parts of a complex nu mber or vector. In the calculation of $\partial \boldsymbol{D}^{*} / \partial b_{\ell}$, the differential of solution $\boldsymbol{x}(t)$ with regard to the parameter $b_{\ell}$ is needed because $\boldsymbol{D}^{*}$ includes $\boldsymbol{x}(t)$ depending on the parameter value. Therefore, the $(i, j)$ th entry of $\partial \boldsymbol{D}^{*} / \partial b_{\ell}$ can be calculated as

$$
\begin{aligned}
\frac{\partial D_{i j}^{*}}{\partial b_{\ell}} & =\sum_{k=1}^{N} \frac{\partial^{2} f_{i}}{\partial x_{j} \partial x_{k}} \cdot \frac{\partial x_{k}^{*}}{\partial b_{\ell}}+\frac{\partial^{2} f_{i}}{\partial x_{j} \partial b_{\ell}} \\
\frac{\partial x_{k}^{*}}{\partial b_{\ell}} & =\left(\boldsymbol{I}-\boldsymbol{D}^{*}\right)^{-1} \cdot \boldsymbol{D}^{*}
\end{aligned}
$$

in which (7) can be derived by differentiating (2) with regard to $b_{\ell}$.

\section{DESIGN OF CONTROLLER}

Consider the situation in which bifurcations of a stable fixed point $\boldsymbol{x}^{*}$ may occur owing to forcible change of $\boldsymbol{a}$ in (1). To simplify the problem, I assume that the values of the system parameters can be changed only at $t=m T(m=1,2, \ldots)$ with $T$ is a sufficiently large integer. I also simplify the notation of $\rho\left(\boldsymbol{D}^{*}\right)$ as $\rho$.

To design a parametric controller to avoid bifurcations of stable fixed points, I consider the minimization problem of an objective function defined by

$$
J(\rho)=\frac{1}{2}(\rho-P(\rho))^{2}
$$

where the map $P: \boldsymbol{R} \rightarrow \boldsymbol{R}$ is defined as

$$
P(\rho)=\left\{\begin{array}{lc}
\rho & \text { if } \rho \leq \hat{\rho} \\
\hat{\rho} & \text { otherwise }
\end{array}\right.
$$

In $(9), \hat{\rho} \in(0,1)$ is a user-defined parameter to detect the approach of para meter values to a bifu rcation point. This means that $J$ takes a positive value only when $\hat{\rho}<\rho<1$, but otherwise $J=0$.

From the aforement ioned as sumption that $\rho$ is differentiable with regard to state variables and system para meters, by using the method of steepest descent, I derived a gradient system of (8), i.e. the parameter regulation of $b_{\ell}$ when $\hat{\rho}<\rho<1$, as

$$
\begin{aligned}
& b_{\ell}((m+1) T)-b_{\ell}(m T) \\
& =-\eta \frac{\partial J(\rho)}{\partial b_{\ell}}=-\eta(\rho-\hat{\rho}) \frac{\partial \rho}{\partial b_{\ell}}
\end{aligned}
$$

where $\eta$ is a user-defined positive parameter.

In summary, the parametric controller I designed detects the approach of system-parameter values to a bifurcation point and updates the value of $b_{\ell}$ on the basis of (10) if $\hat{\rho}<\rho<1$. Therefore, since the value of $\rho$ approaches $\hat{\rho}$ during the period that the parameter regulation in (10) works, the $\rho$ value does not become 1 or more, i.e., it is expected that the proposed controller can avoid bifurcations of stable fixed point.

\section{NUMERICAL EXPERIMENTS}

Is et the parameter values in (10) to $\eta=0.5$ and $\hat{\rho}=0.9$. To show the effectiveness of the designed controller, I carried out numerical experiments for a stable fixed point in the Hénon map defined as

$$
\begin{aligned}
& x_{1}(t+1)=1+x_{2}(t)-a(t) \cdot x_{1}(t)^{2} \\
& x_{2}(t+1)=b(t) \cdot x_{1}(t)
\end{aligned}
$$

When I set the initial parameter values to $a(0)=0.25$ and $b(0)=0.3$, I found a stable fixed point $x^{*}=(1.041,0.312)^{\mathrm{T}}$ with $\rho=0.867$. I preliminarily analyzed the $\rho$ values in a parameter plane and illustrated them as a contour map in Fig. 1. In the left-side parameter region of the contour curve with $\rho=$ 1 , the fixed point is stable and its bifurcation occurs when parameter values are on the contour curve.

On the basis of the preliminary analyzed results, I forcibly changed $a(t)$ as $a((m+1) T)=a(m T)+0.0015$ along the bold blue line from the white circle corresponding to the initial parameter values so that a bifurcation of $\boldsymbol{x}^{*}$ can occur. As the results, the fixed point without parametric control bifurcated on the contour curve with $\rho=1$, and a stable two-periodic point newly occurred at the parameter values.

On the other hand, a bold red curve branches from the blue line and extends toward the lower right along the contour curve with $\rho=0.9$. This red curve represents the trajectories of the 
parameter values with control and illustrated that the proposed controller was able to avoid the bifurcation of the stable fixed point because the red trajectory followed the contour curve with $\rho=0.9$ and did not cross the contour curve with $\rho=1$.

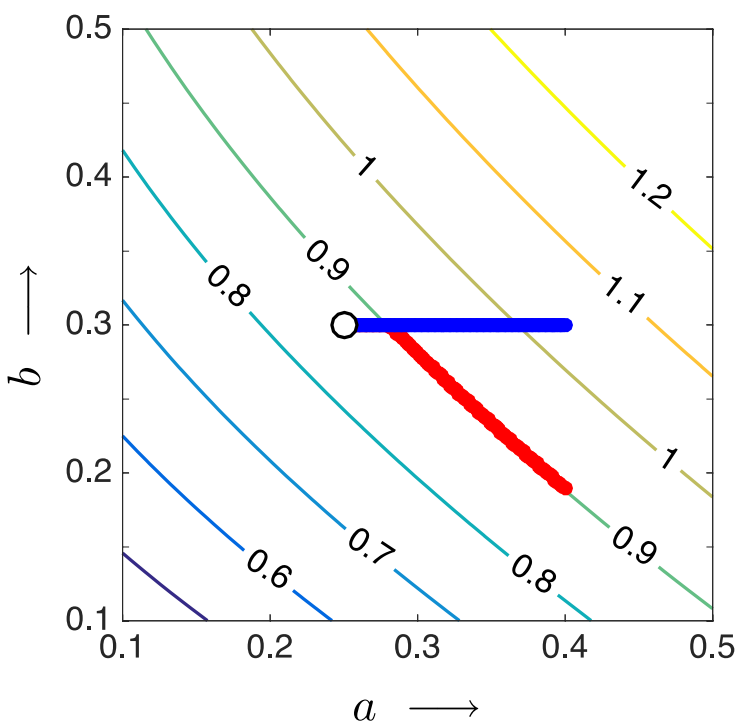

Fig. 1 Experimental results of bifurcation avoidance for stable fixed point using proposed controller

\section{DISCUSSION}

First, I discuss the effectiveness of the designed controller. As seen the blue and red curves in Fig. 1, in the situation that a bifurcation of a stable fixed point can occur, the designed controller detected the approach of parameter values to a bifurcation point by monitoring the $\rho$ value and suppressed the $\rho$ value so that $\rho=\hat{\rho}$ by regulating the value of $b$ during the period that $\hat{\rho}<\rho<1$. As the results, the bifurcation caused by forcible change of $a$ was avoided, and this shows that the proposed controller is effective for the stable fixed point. Here, I note that the proposed controller does not need the results of preliminary analysis to draw the contour map on $\rho$. The map was just drawn to make the effectiveness of the proposed controller easy to understand intuitively.

Next, I discuss the versatility of my approach. The proposed controller, i.e. the parameter regulation in (10), is derived with the method of steepest descent to minimize a quadratic objective function. The approach is generally used to solve optimization problems. From the generality of the objective function and the derivation of (10), this approach can be used for any nonlinear discrete-time dynamical systems.

\section{CONCLUSION}

To avoid inappropriate bifurcations of stable fixed points, which are caused by unexpected change of parameter values, I proposed a parametric controller based on optimizing the spectral radius of the Jacobian matrix. Numerical experiments demonstrated that the proposed controller could be used to avoid a bifurcation of a stable fixed point in the Hénon map.
Moreover, I discussed the effectiveness and versatility of the proposed controller.

\section{ACKNOWLEDGMENT}

This work was supported by JSPS KAKENHI Grant Nu mber JP16K00332.

\section{REFERENCES}

[1] H. Kawakami, "Bifurcation of periodic responses in forced dynamic nonlinear circuits: Computation of bifurcation values of the system parameters," IEEE Trans. Circuits Syst., vol.31,pp. 248-260, 1984.

[2] J. Sun, F. Amellal, L. Glass, and J. Billette, "Alternans and period-doubling bifurcations in atrioventricular nodal conduction," $J$. Theor. Biol., vol. 173,pp.79-91,1995.

[3] K. Fujimoto and K. Aihara, "Bifurcation avoidance control of stable periodic points using the maximum local Lyapunov exponent," Nonlinear Theory and Its Applications, IEICE, vol. 6, pp. 2-14, 2015 .

[4] K. Fujimoto, T. Yoshinaga, T. Ueta, and K. Aihara, "Parametric Control to Avoid Bifurcation Based on Maximum Local Lyapunov Exponent," in Analysis and Control of Complex Dynamical Systems, K. Aihara, J. Imura, and T. Ueta Eds. Tokyo: Springer Japan, 2015, pp.49-55.

[5] K. Fujimoto, T. Otsu, H. Kitajima, and T. Ueta, "Control Technique of Maximum Local Lyapunov Exponent on Stable Periodic Solution in Continuous-Time Non-Autonomous Dynamical Systems," International Journal of Computing, Communication and Instrumentation Engineering,vol.3, pp. 349-353, 2016.

[6] Y. Kima, D. Gub, and I. Postlethwaite, "Spectral radius minimization for optimal average consensus and output feedback stabilization," Automatica, vol. 45, pp. 1379-1386, 2009.

[7] M. Hénon, "A two-dimensional mapping with a strange attractor," Commun. Math. Phys., vol. 50, pp. 69-77, 1976. 